\title{
The Cell Death in CMS Plants
}

\author{
Ling Huang, Chen Zhang, Ming Fang, Hao Xu, and Moju Cao
}

\begin{abstract}
Reactive oxygen species (ROS)-mediated PCD was found to occur in CMS lines of many species. The excess production of ROS is probably caused by the decrease of the activity of ROS-scavenging enzymes in CMS plants at the abortion peak. Why antioxidant enzyme activities decline? Mostly, the ascorbate-glutathione cycle of mitochondria is blocked by the depletion of ATP and NADH which would result from mitochondrial dysfunction induced by the expression of mitochondrial chimeric open-reading frames (ORFs). Moreover, the interaction between ROS and some signaling molecules, such as ethylene, attenuated the induction of the cyanide-resistant respiration, which exacerbates the accumulation of the ROS. Under oxidative stress, cellular protein especially membrane protein and DNA damage occur, and thus lead to the cell death. The characteristic feature of the programmed cell death (PCD) in CMS plants may be that it can be blocked by a matching nuclear pathway that restores normal regulation. We will discuss the central role of the mitochondrion in the whole pathways of the cell death in CMS plants.
\end{abstract}

Index Terms-Cytoplasmic male sterility, Mitochondri-al, Programmed cell death.

\section{INTRODUCTION}

Cytoplasmic male sterility (CMS) is a maternally inherited trait in higher plants that results in the inability of the mature plant to produce functional pollen. CMS results from the expression of chimeric ORFs encoded by the mitochondrial genome, which can be reversed by its own specific matching nuclear restorer genes [1]. At least 14 mitochondrial ORFs that determine CMS have been characterized, which comprise segments derived from mitochondrial gene-coding and gene-flanking sequences and from sequences of unknown origin [1], [2]. ORFs interact with the anther-specific factor such as metabolites or gene products, which induce mitochondrial dysfunction [3]. Cell death occurs when mitochondrial function and ATP levels are insufficient to support plant development. Cell death in mitochondria is not only through changes in ATP production but also accumulation of ROS which increases under conditions of mitochondrial dysfunction [4]. The association of PCD with mitochondria-mediated CMS provides some of the first evidence of mitochondrial involvement in plant PCD pathways [5].

Manuscript received September 1, 2011; revised November 24, 2011.

L. Huang, C. Zhang, M. Fang, and H. Xu are with the Maize Research Institute of Sichuan Agricultural University/Key Laboratory of Crop Genetic Resource and Improvement, Ministry of Education, Ya'an 625014, Sichuan, China (e-mail: b1983429@126.com, 513657334@qq.com, 79145 0070@qq.com,448553819@qq.com, respectively)

M. J. Cao (Corresponding author), is with the Maize Research Institute of Sichuan Agricultural University/Key Laboratory of Crop Genetic Resource and Improvement, Ministry of Education/ Key Laboratory of maize biology and Genetic Breeding on Southwest, Ministry of Agriculture, Ya`an 625014, Sichuan, China (e-mail:mojupp@163.com).

\section{CMS-AsSOCIATED CHIMERIC ORFS ARE OFTEN TOXIC}

In previous studies, mitochondrial chimeric genes have generally been shown to encode peptides that are lethal to $E$. coli. The most extensively analyzed CMS system is probably the CMS-T maize. In CMS-T, mitochondrial chimeric gene T-urf13 encodes a $13 \mathrm{kDa}$ polypeptide (URF13) which strongly inhibits E. coli growth [6]. A few other CMS-associated genes (sunflower orf522, Brassica Ogura radish orf138 and BT-rice orf79) have also been shown to encode the toxic protein [7]-[9]. In addition, the toxicity of this kind of peptide in eukaryotic cell has also been studied. For example, orfH79 is a mitochondria chimeric gene being responsible for the CMS trait in Honglian (HL) rice. Weakly expressed ORFH79 strongly inhibits the growth of yeast cells [10]. More recently, a number of transgenic experiments have also confirmed the toxicity of ORF. After introducing the BT-rice orf79 gene into Taichung 65 carrying normal cytoplasm, it is revealed that ORF79 is toxic to plant regeneration when expresses as a fusion with mitochondrial targeting sequence of the ATPase subunit [11]. The CMS-associated mitochondrial protein in petunia, ORF129 results in male sterility in transgenic tobacco plants [12]. It is reported that the expression of sunflower cytoplasmic male sterilityassociated open reading frame, orfH522 induces male sterility in transgenic tobacco plants [13].

\section{CMS-ASSOCIATED CHIMERIC ORFS AND TAPETUM DISINTEGRATION}

What kind of the relationship between CMS-ass- ociated ORFs and the PCD? The defect in mitochondrial function is caused by a CMS associated ORFs, its ultimate result may be programmed cell death (PCD) of either the tapetal layer or sporogenous cells. In most CMS plants, morphological studies suggests that mitochondria often cause cytoplasmic male sterility by triggering programmed cell death of the tapetum that nurtures pollen mother cells [14]. The maize mitochondrial gene T-urf13 encodes a toxin protein that results in early tapetal degeneration soon after microspore meiosis [15]. In PET1-CMS sunflower, the expression of mitochondrial chimeric ORF causes a classical PCD in tapetal tissues, which includes oligonucleosomal cleavage of nuclear DNA, separation of chromatin into delineated masses, and partial release of cytochrome c into the cytosol of tapetal cells [16]. In other male sterility plants, premature tapetum degeneration also causes the abortive of pollen, such as the thermosensitive and photoperiod-sensitive malesterile rice [17],[18].

One of the biochemical features of PCD in CMS plant is that each mitochondrial pathway for interfering with the regulation of PCD can be blocked by a matching nuclear 
pathway that restores normal regulation [19]. Microsporogenesis in restored maize CMS-T (A239 $R f$ ) plants is quite different from the male sterile plants, while it is not significantly different from that in normal plants [20]. Similar PCD pathways are blocked by matching nuclear restorer genes in cotton cytoplasmic male sterility and maize CMS-S [21], [22].

However, the mechanism of blocking process is not known so far. It is difficult to establish whether the nuclear restorer genes regulated the expression of antiapoptosis genes or blocked the expression of CMS-associated mtDNA which subsequentially inhibited mitochondrial apoptosis pathways. As far as we know, there is little evidence which is found in CMS-S maize [22]. The transcripts of antiapoptosis genes accumulated in the development of $\mathrm{S}-(R f 3)$ gametophytes which carry S-type sterile cytoplasm and nuclear restorer gene ( $R f 3 R f 3)$. But it was not enough to decide that the nuclear restorer genes induced the expression of antiapoptosis genes which blocked the PCD. After all, the accumulation of ROS is not radically reduced.

Why does the PCD mostly occur in tapetal rather than other tissues in male sterility? Microscopic evidence suggests that mitochondrial activities are important in the tapetal layer. During early development of normal maize anthers, the number of maize mitochondria increases about 20 times in sporogenous tissues and 40 times in tapetal cells [23], respectively. A significant increase in the number of mitochondria in pollen reflects a substantial increase of energy demand. Tapetal tissue is particularly sensitive to mitochondrial aberrations and is usually the only tissue that exceeds the threshold for showing signs of mitochondria-induced failure. Tapetal cells as the key pollen coat, precocious tapetal deterioration can also result in male sterility. Presumably, critical nutrient resources would not support for proper microspore development in the absence of a differentiated tapetum. Moreover, some signaling genes are preferentially expressed in tapetal cells, such as ethylene signaling genes [24].

\section{HOW DO THE CMS-AsSOCIATED ORFS WORK?}

The expression of ORFs is thought to interfere in the normal function of mitochondria and lead to the abnormal development of tapetum or microspores during reproductive stage. Mitochondrion provides energy for all kinds of activities in cells through oxidation phosphorylation. Meanwhile, mitochondrion is also a major source of ROS formation and it may participate in the oxidative burst in plants which trigger cell death [25]. In order to repress mitochondrial cell-death signaling pathways in all except anthers, regulators of ROS-mediated signaling triggered cell death [5]. Numerous studies suggested that pollen abortion of the sterile plants is associated with PCD, such as PET1-CMS in sunflower, Honglian (HL)-CMS line of rice, cotton cytoplasmic male sterility, CMS-T and CMS-S of maize [15], [16], [21], [22], [26]. Most PCD is related with excessive production of ROS and lower ROS-scavenging enzymic activities in CMS plants at the abortion peak.

In plant cells, the mitochondrial electron transport chain is a major site of ROS production. Complex I is a major site of ROS production, together with complex III. It has been reported that mitochondrial ROS is significantly higher in tobacco CMS II which lack of complex I compared with the wild type [27]. The inhibition of complex I by rotenone induces increase of mitochondrial ROS in Arabidopsis [28]. In several CMS plants, such as tobacco CMS I and II and CMS stem mustard (Brassica juncea) have been proved to be associated with the abnormal NADH ubiquinone oxidoreductase gene [29]-[31]. The generation rate of ROS, e.g. O2-, $\mathrm{H}_{2} \mathrm{O}_{2}$, in sterile line is higher than that in maintainer line [21], [26], [32].

In the sterile anther mitochondria, the production of ROS significantly increased, while the activities of ROS-scavenging enzymes remarkably reduced, which might break the balance between ROS production and elimination. However, the activities of antioxidant enzymes in sterile line are not lower than those in the corresponding maintainer at any time. ROS-scavenging enzymes include SOD, APX, GR, DHAR and MDHAR, GSH and ascorbate acid and so on. At the stage of preliminary abortion, ROS begins to accumulate, which serves as the signal to up-regulate the transcription of antioxidant enzymes encoded in nuclear genome. At the peak of abortion, the activities of the enzymes are lower in CMS line than those in its maintainer line. It is not clear why antioxidant enzymes activities decrease in CMS plant. The possibility might be the oxidative damage to the structure and active sites of these proteins [32]. However, it may be not the key factor. The potential mechanism is shown in Fig. 1 based on the previous studies.

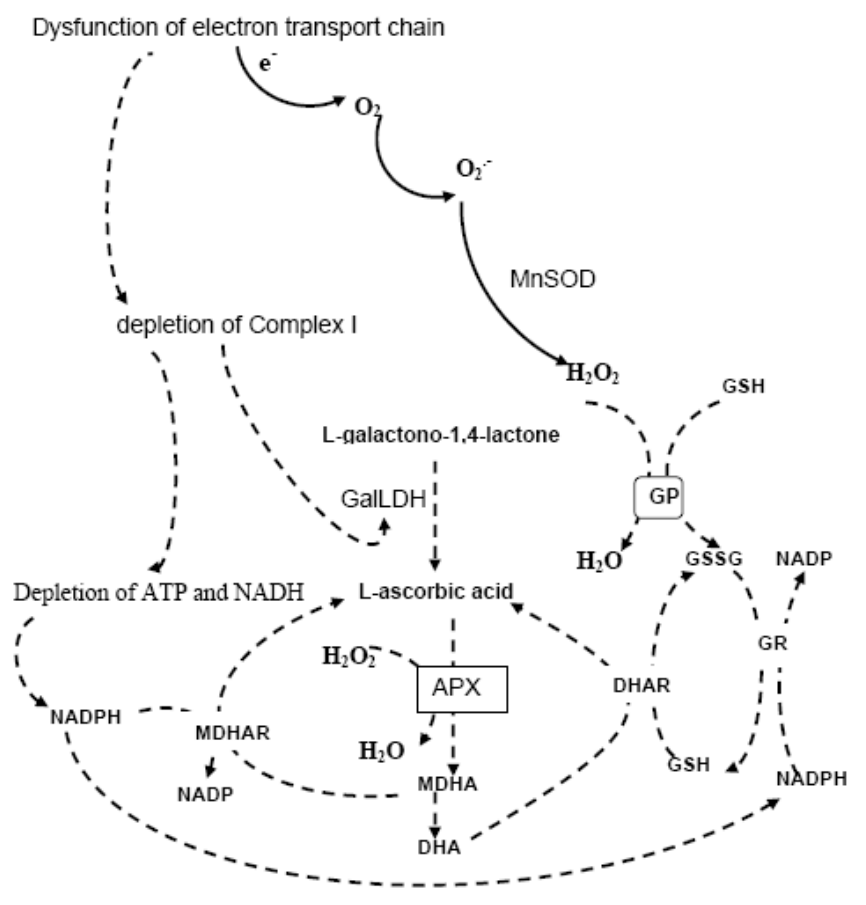

Fig. 1. The hypothesis of reducing ROS-scavenging enzymic activities in CMS at the peak of abortion. GalLDH: L-galactono-1,4-lactone dehydrogenase; APX: Aseorbate peroxidase; MDHAR: Monodehydroascorbate reductase; DHAR: Dehydroascorbate reductase; GR: glutathione reductase; GP: glutathione peroxidase; GSSG: oxidative glutathione; GSH: reduced Glutathione.

A single electron escapes from NADH-ubiquinone oxidoreductase. Molecular oxygen becomes superoxide anion radical (O2.-) by a single electron transfer to molecular oxygen and then O2.- is converted to $\mathrm{H}_{2} \mathrm{O}_{2}$ by $\mathrm{Mn}$ superoxide dismutase (MnSOD). $\mathrm{H}_{2} \mathrm{O}_{2}$ is detoxified by 
reduced glutathione (GSH) changes into oxidized glutathione GSSG. The balance between GSH and GSSG depends on the supply of NADPH. The depletion of ATP and $\mathrm{NAD}^{+}-\mathrm{NADH}$ pool are testified in the CMS-HL rice [32]. With the decrease of NADH and ATP, NADPH level reduces. The increased GSSG impairs the scavenging of $\mathrm{H}_{2} \mathrm{O}_{2}$, and then MnSOD activity is reversely inhibited. Accordingly, the expression of SOD is down-regulated during the development of microspores in HL-CMS line [32]. Besides, the ascorbate-glutathione cycle of mitochondria is another important system in resisting ROS [33]. The final enzyme of ascorbate synthesis in plants, converting galactolactone to ascorbate, is physically associated with complex I [34], [35]. The abnormal expression of complex I reduces the synthesis of ascorbate, which affects the ascorbate-glutathione cycle in mitochondria. Complex I would most likely regulate the synthesis of ascorbate through L-galactono-1, 4-lactone dehydrogena se (GalLDH). GalLDH is an integral protein of the inner mitochondrial membrane, which catalyzes L-galactono-1, 4-lactone converted to L-ascorbic acid [36]. The decrease of L-ascorbic acid attenuates the activities of APX, GR, DHAR, MDHAR and GSH. Therefore, the expression of APX, GR, DHAR, MDHAR and SOD are down-regulated during the development of microspores in CMS line [21], [32].

\section{INTERACTION BETWEEN ETHYLENE AND ROS TO CONTROL PCD}

The ROS-dependent cell death is regulated by the plant hormones. As a plant hormone, ethylene is an important molecular in regulating ROS-dependent cell death [37],[38]. It can stimulate PCD in floral organs [39] and induce male sterility in a number of species including wheat [40], millet [41], and Brassica [42]. ROS regulates the synthesis of ethylene [43]. In the ethylene synthesis pathway, 1-aminocyclopropane-1-carboxylic acid (ACC) synthase activity, ACC content and ethylene emission are all required for ROS accumulation [44]. On the contrary, the increased ROS production in the mitochondria could also be perturbed through changes in ethylene synthesis and sensitivity. Thus, high ethylene synthesis attenuates induction of the alternative oxidase (AOX) or cyanide-resistant respiration [45]. It has been proved that many CMS lines lacked cyanide-resistant respiration [46], which can increase ROS production [47]. Consistent with this, tobacco cells lacking AOX or cyanide-resistant respiration increased PCD in response to $\mathrm{H}_{2} \mathrm{O}_{2}$ [48]. Furthermore, the downregulation of AOX or pyruvate dehydrogenase in the tobacco tapetum results in microspore death [49]. Ethylene signaling genes are preferentially expressed in rice tapetal cells, especially during the tetrad and unicellular stages [24], which suggest that ROS may firstly accumulate in tapetal cells, and trigger PCD in CMS anthers. It may be also the reason for the PCD firstly occurs in tapetal rather than other tissues, as described in Capsicum annuum, Brassica napus [50],[51].

\section{OCCURRENCE OF PCD IN CMS PLANTS}

When ROS generation exceeds the cellular capacity to scavenge, cellular proteins especially membrane protein and DNA damage occur, and thus lead to the cell death (Fig. 2). Under oxidative stress, mitochondrial membrane is susceptible to oxidative damage by ROS. The loss of mitochondrial membrane permeability is thought to be the center for PCD mediated by ROS. The microscopic analysis of CMS plants in many species has indicated that disruption of mitochondrial integrity in the tapetal layer is the first sign of abnormal development and thus may be the cause of microspore abortion [52],[53]. The voltage-dependent anion channel (VDAC) is an important complex of the mitochondrial permeability transition pore (PTP) [54]. $\mathrm{NADH}$ regulates the gating of VDAC [55],[56]. Cytochrome C, DNase and apoptotic factors could all be released by opening PTP, which can trigger PCD [57]-[59]. In CMS plant, the mitochondrial genomic DNA and nuclear DNA are both degraded by DNase [16], [26], [32].

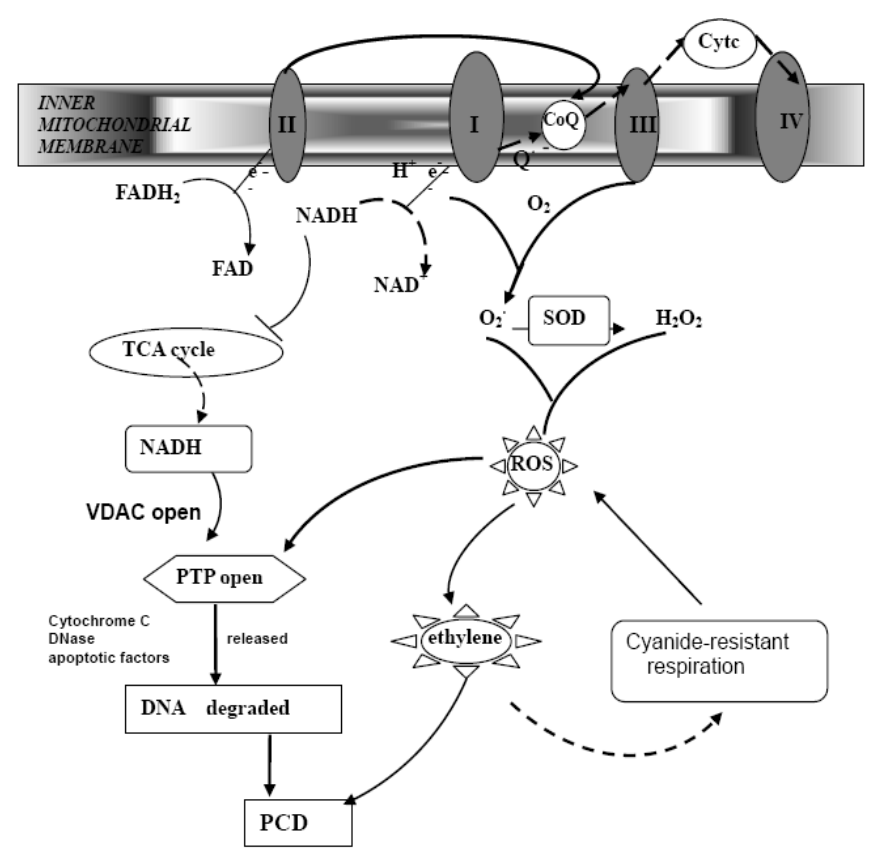

Fig. 2. Occurrence of PCD in CMS plants. In CMS plant cells, the expression of ORFs is thought to interfere in the normal function of the mitochondrial electron transport chain (ETC) and TCA cycle. Mostly, dysfunction of the mitochondria causes the depletion of ATP, NADH and accumulation of ROS. On the one hand, depletion of NADH causes the VDAC open which forms the mitochondrial permeability transition pore (PTP). On the other hand, when ROS generation exceeds the cellular capacity to detoxify them, ROS can induce the PTP open. Moreover, ROS inducts synthesis of ethylene. High ethylene synthesis attenuated induction of the AOX or cyanide-resistant respiration. The lack of cyanide-resistant respiration induction caused increases ROS production. And then, Cytochrome C, DNase and apoptotic factors release. DNA is hydrolyzed by DNase, leading to the PCD at last.

\section{CONCLUSIONS}

In this review, we have sketched out the major metabolism pathways for the ROS production and trigger PCD in CMS plants. The common features of both biotic and abiotic stress responses are transient oxidative burst and subsequent temporary shift in the intracellular redox state [60], [61]. However, PCD in CMS plants is different from that in abiotic stress conditions such as temperature extremes, drought, or salt stress. In CMS plants, PCD is triggered by the mitochondrial dysfunction induced by the interaction between ORFs and anther-specific factors, which 
could be blocked by a matching nuclear pathway that restores normal regulation. Currently, while there is incontrovertible that such pathway does exist, but we do not know how such a pathway would work. Analysis the characterization of this pathway will lead to a better understanding of cell death in CMS plants.

\section{REFERENCE}

[1] M. Hanson and S. Bentolila, "Interactions of mitochondrial and nuclear genes that affect male gametophyte development," The Plant Cell, vol. 16, pp. S154-169, June 2004.

[2] C. Chase and S. Gabay-Laughnan, "Cytoplasmic male sterility and fertility restoration by nuclear genes," Molecular Biology and Biotechnology of Plant Organelles, vol. 3, pp. 593-621, 2004.

[3] R. Flavell, "A model for the mechanism of cytoplasmic male sterility in plants, with special reference to maize,” Plant Science Letters, vol. 3, pp. 259-263, October1974.

[4] M. Bras, B. Queenan, and S. Susin, "Programmed cell death via mitochondria: different modes of dying," Biochemistry (Moscow). vol. 70, pp. 231-239, September 2005.

[5] C. Chase, "Cytoplasmic male sterility: a window to the world of plant mitochondrial-nuclear interactions," Trends in Genetics, vol. 23, pp. 81-90, February 2007.

[6] R. Dewey, J. Siedow, D. Timothy, and C. Levings 3rd, "A 13-kilodalton maize mitochondrial protein in $E$. coli confers sensitivity to Bipolaris maydis toxin,” Science, vol. 239, pp. 293, January 1988.

[7] Z. Wang, Y. Zou, X. Li, Q. Zhang, L. Chen, H. Wu, D. Su, Y. Chen, and J. Guo, et al, "Cytoplasmic male sterility of rice with boro II cytoplasm is caused by a cytotoxic peptide and is restored by two related PPR motif genes via distinct modes of mRNA silencing,” The Plant Cell, vol. 18, pp. 676-687, March 2006.

[8] Y. Duroc, C. Gaillard, S. Hiard, M. Defrance, G. Pelletier, and F. Budar, "Biochemical and functional characterization of ORF138, a mitochondrial protein responsible for Ogura cytoplasmic male sterility in Brassiceae,” Biochimie, vol. 87, pp.1089-1100, December 2005.

[9] S. Nakai, D. Noda, M. Kondo, and T. Terachi, "High-level expression of a mitochondrial orf 522 gene from the male-sterile sunflower is lethal to E. coli,” Breeding Science, vol. 45, pp.233, 1995.

[10] X. Peng, F. Li, S. Li, and Y. Zhu, "Expression of a mitochondrial gene orfH79 from the CMS-HongLian rice inhibits Saccharomyces cerevisiae growth and causes excessive ROS accumulation and decrease in ATP," Biotechnology letters, vol. 31, pp. 409-414, November 2009.

[11] H. Kojimaa, T. Kazama, S. Fujii, and K. Toriyama, "Cytoplasmic male sterility-associated ORF79 is toxic to plant regeneration when expressed with mitochondrial targeting sequence of ATPase $\gamma$ subunit," Plant Biotechnology, vol. 27, pp. 111-114, September 2010.

[12] M. Yamamoto, H. Shinada, Y. Onodera, C. Komaki, T. Mikami, and T Kubo, "A male sterility-associated mitochondrial protein in wild beets causes pollen disruption in transgenic plants," The Plant Journal, vol. 54, pp. 1027-1036, June 2008.

[13] N. Nizampatnam, H. Doodhi, Y. Kalinati Narasimhan, S. Mulpuri, and D. Viswanathaswamy, "Expression of sunflower cytoplasmic male sterility-associated open reading frame, orf H522 induces male sterility in transgenic tobacco plants,” Planta, vol. 229, pp. 987-1001, January 2009.

[14] H. Wu and A. Cheung, "Programmed cell death in plant reproduction," Plant molecular biology, vol. 44, pp. 267-281, 2000.

[15] P. Schnable and R. Wise. The molecular basis of cytoplasmic male sterility and fertility restoration,” Trends in Plant Science, vol. 3, pp. 175-180, May 1998.

[16] J. Balk and C. Leaver, "The PET1-CMS mitochondrial mutation in sunflower is associated with premature programmed cell death and cytochrome c release,” The Plant Cell, vol. 13, pp. 1803, August 2001.

[17] Y. Shi, S. Zhao, and J. Yao, "Premature tapetum degeneration: a major cause of abortive pollen development in photoperiod sensitive genic male sterility in rice,” Journal of Integrative Plant Biology, vol. 51, pp. 774-781, August 2009.

[18] S. Ku, H. Yoon, H. Suh, and Y. Chung, "Male-sterility of thermosensitive genic male-sterile rice is associated with premature programmed cell death of the tapetum," Planta, vol. 217, pp. 559-565, April 2003.
[19] S. Frank and C. Barr, "Programmed cell death and hybrid incompatibility," Journal of Heredity, vol. 94, pp.181, 2003,

[20] C. Colhoun and M. Steer, "Microsporogenesis and the mechanism of cytoplasmic male sterility in maize," Annals of Botany, vol. 48, pp. 417, 1981

[21] P. Jiang, X. Zhang, Y. Zhu, W. Zhu, H. Xie, and X. Wang, "Metabolism of reactive oxygen species in cotton cytoplasmic male sterility and its restoration," Plant cell reports, vol. 26, pp.1627-1634, April 2007.

[22] Z. Zhang, W. Tang, F. Zhang, and Y. Zheng, "Fertility restoration mechanisms in S-type cytoplasmic male sterility of maize (Zea mays L.) revealed through expression differences identified by cDNA microarray and suppression subtractive hybridization," Plant Molecular Biology Reporter, vol. 23, pp. 17-38, March 2005.

[23] H. Warmke and S. Lee, "Pollen Abortion in T Cytoplasmic Male-Sterile Corn (Zea mays): A Suggested Mechanism,” Science, vol. 200, pp. 561, May 1978.

[24] K. Hirano, K. Aya, T. Hobo, H. Sakakibara, M. Kojima, R. Shim, Y. Hasegawa, M. Ueguchi-Tanaka, and M. Matsuoka, "Comprehensive transcriptome analysis of phytohormone biosynthesis and signaling genes in microspore/pollen and tapetum of rice," Plant and Cell Physiology, vol. 49, pp.1429, August 2008.

[25] B. Mignotte and J. Vayssiere, "Mitochondria and apoptosis," European Journal of Biochemistry, vol. 252, pp. 1-15, February 1998.

[26] S. Li, C. Wan, J. Kong, Z. Zhang, Y. Li, and Y. Zhu, "Programmed cell death during microgenesis in a Honglian CMS line of rice is correlated with oxidative stress in mitochondria," Functional Plant Biology, vol. 31, pp. 369-376, 2004.

[27] G. Vidal, M. Ribas-Carbo, M. Garmier, G. Dubertret, A. Rasmusson, C. Mathieu, C. Foyer, and R. De Paepe, "Lack of respiratory chain complex I impairs alternative oxidase engagement and modulates redox signaling during elicitor-induced cell death in tobacco," The Plant Cell, vol. 19, pp.640, February 2007.

[28] M. Garmier, A. Carroll, E. Delannoy, C. Vallet, D. Day, I. Small, and A. Millar, "Complex I dysfunction redirects cellular and mitochondrial metabolism in Arabidopsis," Plant physiology, vol. 148, pp.1324, November 2008.

[29] S. Gutierres, M. Sabar, C. Lelandais, P. Chetrit, P. Diolez, H. Degand, M. Boutry, F. Vedel, Y. De Kouchkovsky, and R. De Paepe, "Lack of mitochondrial and nuclear-encoded subunits of complex I and alteration of the respiratory chain in Nicotiana sylvestris mitochondrial deletion mutants," Proc Natl Acad Sci USA, vol. 94, pp. 3436, April 1997.

[30] M. Pla, C. Mathieu, R. De Paepe, P. Chétrit, and F. Vedel, "Deletion of the last two exons of the mitochondrial nad7 gene results in lack of the NAD7 polypeptide in a Nicotiana sylvestris CMS mutant," Molecular and General Genetics MGG, vol. 248, pp.79-88, March 1995.

[31] J. Yang, M. Zhang, and J. Yu, "Mitochondrial nad2 gene is co-transcripted with CMS-associated orfB gene in cytoplasmic male-sterile stem mustard (Brassica juncea)," Molecular biology reports, vol. 36, pp. 345-351, November 2009.

[32] C. Wan, S. Li, L. Wen, J. Kong, K. Wang, and Y. Zhu, "Damage of oxidative stress on mitochondria during microspores development in Honglian CMS line of rice," Plant cell reports, vol. 26, pp. 373-82, October 2007.

[33] A. Jimenez, J. Hernandez, L. del Rio, and F. Sevilla, "Evidence for the presence of the ascorbate-glutathione cycle in mitochondria and peroxisomes of pea leaves," Plant physiology, vol. 114, pp. 275, May 1997.

[34] J. Heazlewood, K. Howell, and A. Millar, "Mitochondrial complex I from Arabidopsis and rice: orthologs of mammalian and fungal components coupled with plant-specific subunits," Biochimica et Biophysica Acta (BBA)-Bioenergetics, vol. 1604, pp.159-169, July 2003.

[35] A. Millar, V. Mittova, G. Kiddle, J. Heazlewood, C. Bartoli, F. Theodoulou, and C. Foyer, "Control of ascorbate synthesis by respiration and its implications for stress responses," Plant physiology, vol. 133, pp.443, October 2003.

[36] V Valpuesta, M Botella, "Biosynthesis of L-ascorbic acid in plants: new pathways for an old antioxidant,” Trends in Plant Science, vol. 9, pp. 573-577, December 2004.

[37] A. Gunawardena, "Programmed cell death and tissue remodelling in plants,” Journal of experimental botany, vol. 59, pp. 445, 2008.

[38] B. Rubinstein, "Regulation of cell death in flower petals," Plant molecular biology, vol. 44, pp. 303-318, 2000.

[39] H. Rogers, "Programmed cell death in floral organs: how and why do flowers die?” Annals of Botany, vol. 97, pp.309, January 2006.

[40] W. Hughes, J. Bodden, and S. galanopoulou, "The effect of sowing density and application of gibberellic acid on male sterility and ear 
emergence in ethephon-treated field-grown wheat,” Annals of Applied Biology, vol. 88, pp. 313-319, March 1978.

[41] R. Thakur and V. Rao, "Effectiveness of ethrel as a male gametocide in pearl millet and its influence on ergot,” Plant Breeding, vol. 101, pp. 107-113, September 1988.

[42] S. Banga and K. Labana, "Production of F1 hybrids using ethrelinduced male sterility in Indian mustard (Brassica juncea (L.) Coss.)," The Journal of Agricultural Science, vol. 101, pp. 453-455, 1983.

[43] M. Samuel and B. Ellis, "Double jeopardy: both overexpression and suppression of a redox-activated plant mitogen-activated protein kinase render tobacco plants ozone sensitive,” The Plant Cell, vol. 14, pp. 2059, 2002.

[44] W. Moeder, C. Barry, A. Tauriainen, C. Betz, J. Tuomainen, M. Utriainen, D. Grierson, H. Sandermann, C. Langebartels, and J. Kangasjrvi, "Ethylene synthesis regulated by bi-phasic induction of ACC synthase and ACC oxidase genes is required for $\mathrm{H}_{2} \mathrm{O}_{2}$ accumulation and cell death in ozone-exposed tomato," Plant Physiol, vol. 130, pp. 1918-1926, September 2002.

[45] K. Overmyer, M. Brosché, and J. Kangasjärvi, "Reactive oxygen species and hormonal control of cell death,” Trends in Plant Science, vol. 8, pp. 335-42, July 2003.

[46] M. Musgrave, J. Antonovics, and J. Siedow, "Is male-sterility in plants related to lack of cyanide-resistant respiration in tissues?" Plant Science, vol. 44, pp. 7-11, January 1986.

[47] D. Maxwell, Y. Wang, and L. McIntosh, "The alternative oxidase lowers mitochondrial reactive oxygen production in plant cells," Proc Natl Acad Sci USA, vol. 96, pp. 8271, July 1999.

[48] C. Robson and G. Vanlerberghe, "Transgenic plant cells lacking mitochondrial alternative oxidase have increased susceptibility to mitochondria-dependent and-independent pathways of programmed cell death,” Science's STKE, vol. 129, pp. 1908, August 2002.

[49] R. Yui, S. Iketani, T. Mikami, and T. Kubo, "Antisense inhibition of mitochondrial pyruvate dehydrogenase E1 a subunit in anther tapetum causes male sterility,” The Plant Journal, vol. 34, pp. 57-66, April 2003.

[50] H. Horner Jr, “A comparative light-and electron-microscopic study of microsporogenesis in male-fertile and cytoplasmic male-sterile sunflower (Helianthus annuus)," American Journal of Botany, 745-759, July 1977.

[51] I. Grant, W. Beversdorf, and R. Peterson, “A comparative light and electron microscopic study of microspore and tapetal development in male fertile and cytoplasmic male sterile oilseed rape (Brassica napus)," Canadian Journal of Botany, vol. 64, pp.1055-1068, 1986.

[52] K. Laser and N. Lersten, "Anatomy and cytology of microsporogenesis in cytoplasmic male sterile angiosperms," The Botanical Review, vol. 38, pp.425-454, April 1972.

[53] M. Kaul, "Male sterility in higher plants," Springer Verlag: Berlin. vol. 1005, pp. 116-117, 1987.

[54] M. Crompton, "The mitochondrial permeability transition pore and its role in cell death,” Biochemical Journal, vol. 341, pp. 233, July 1999.

[55] M. Zizi, M. Forte, E. Blachly-Dyson, and M. Colombini, "NADH regulates the gating of VDAC, the mitochondrial outer membrane channel," Journal of Biological Chemistry, vol. 269, pp. 1614 January 1994

[56] A. Lee, X. Xu, and M. Colombini, "The role of pyridine dinucleotides in regulating the permeability of the mitochondrial outer membrane," Journal of Biological Chemistry, vol. 271, pp. 26724, October 1996

[57] J. Lemasters, "Dying a thousand deaths: redundant pathways from different organelles to apoptosis and necrosis," Gastroenterology, vol. 129, pp. 351-360, July 2005.

[58] D. Green and G. Kroemer, "The pathophysiology of mitochondrial cell death,” Science, vol. 305, pp.626, July 2004.

[59] J. Balk, S. Chew, C. Leaver, and P. McCabe, "The intermembrane space of plant mitochondria contains a DNase activity that may be involved in programmed cell death,” The Plant Journal, vol. 34, pp. 573-583, June 2003.

[60] R. Mittler, S. Vanderauwera, M. Gollery, and F. Van Breusegem, "Reactive oxygen gene network of plants," Trends in Plant Science, vol. 9, pp. 490-498, October 2004

[61] J. Dat, S. Vandenabeele, E. Vranova', M. Van Montagu, D. Inze', and F. Van Breusegem, "Dual action of the active oxygen species during plant stress responses," Cellular and molecular life sciences, vol. 57, pp.779-795, January 2000. 